\title{
MATHEMATICAL MODELING OF THERMOFRICTIONAL MILLING PROCESS USING ANSYS WB SOFTWARE
}

\author{
K.T. SHEROV ${ }^{1}$, M.R. SiKhIMBAYEV ${ }^{2 *}$, A.K. SHEROV ${ }^{1}$, \\ B.S. DONENBAYEV ${ }^{1}$, A.K. RAKISHEV ${ }^{1}$, A.B. MAZDUbai ${ }^{1}$, \\ M.M. Musayev ${ }^{1}$, A.M. Abeuova ${ }^{1}$ \\ ${ }^{1}$ Karaganda State Technical University, 56 B. Mira St., 100027 Karaganda, \\ Kazakhstan \\ ${ }^{2}$ Karaganda Economic University, 9 Akademicheskaya St., 100009 \\ Karaganda, Kazakhstan
}

[Received 12 January 2017. Accepted 26 June 2017]

\begin{abstract}
This article presents ANSYS WB-based mathematical modelling of the thermofrictional milling process, which allowed studying the dynamics of thermal and physical processes occurring during the processing. The technique used also allows determination of the optimal cutting conditions of thermofrictional milling for processing various materials, in particular steel 40CN2MA, 30CGSA, 45, 3sp. In our study, from among a number of existing models of cutting fracture, we chose the criterion first proposed by prof. V. L. Kolmogorov. In order to increase the calculations performance, a mathematical model was proposed, that used only two objects: a parallelepiped-shaped workpiece and a cutting insert in the form of a pentagonal prism. In addition, the work takes into account the friction coefficient between a cutting insert and a workpiece taken equal to $0.4 \mathrm{~mm}$. To determine the temperature in the subcontact layer of the workpiece, we introduced the coordinates of nine characteristic points with the same interval in the local coordinate system. As a result, the temperature values were obtained for different materials at the studied points during the cutter speed change. The research results showed the possibility of controlling thermal processes during processing by choosing the optimum cutting modes.
\end{abstract}

KEY WORDS: Thermofrictional milling, cutting insert, friction coefficient, temperature, subcontact layer, fracture model, finite element mesh, flat surface, work hardening, mathematical model, thermal conductivity.

\section{RELEVANCE OF THE RESEARCH}

Thermofrictional processing is a type of thermomechanical technology for metal processing with the resulting heating in the cutting zone, due to friction at the point of

${ }^{*}$ Corresponding author e-mail: smurat@yandex.ru 
contact of a workpiece and a tool moving at high speed [1]. Traditional thermofrictional technology is based on softening the processed material in the cutting zone due to high sliding friction rate.

The higher the speed, the more heat accumulates at the contact. The material to be processed, hence, is subjected to more softening and cutting disc retains its strength properties due to minimized stay of a particular part of the disc side in the contact. However, an excessive increase in the speed leads to stricter requirements for the tooling. Thus, the average cost of a machine at an increase in speed from $V=30 \mathrm{~m} / \mathrm{s}$ to $V>70 \mathrm{~m} / \mathrm{s}$ rises $5-7$ times, and the electric power consumption increases likewise (machines for thermofrictional treatment have $22-40 \mathrm{~kW}$ engines). Consequently, designing a resource-saving technology of low-speed thermofrictional processing is a highly relevant task.

The authors are currently conducting a government-funded research "Designing a special machine, allowing the pulsed cooling supply and replacement of a hard-alloy cutting tool with a tool made of structural steel, during thermofrictional cutting of metal workpieces" (Contract No. 723 of 12.02.2015 and No. 336-13 of 13.05.2016). This machine has functions of resource-saving thermofrictional processing at low cutting speeds [2-4]. One of these modes is thermofrictional milling of flat surfaces with pulsed cooling. The research results showed positive results in terms of qualitative parameters of processing various materials $[5,6]$.

Figure 1 shows the process of thermofrictional milling of 30CGSA steel. Cutting tool is an end mill equipped with plates of HARDOX 450 steel.

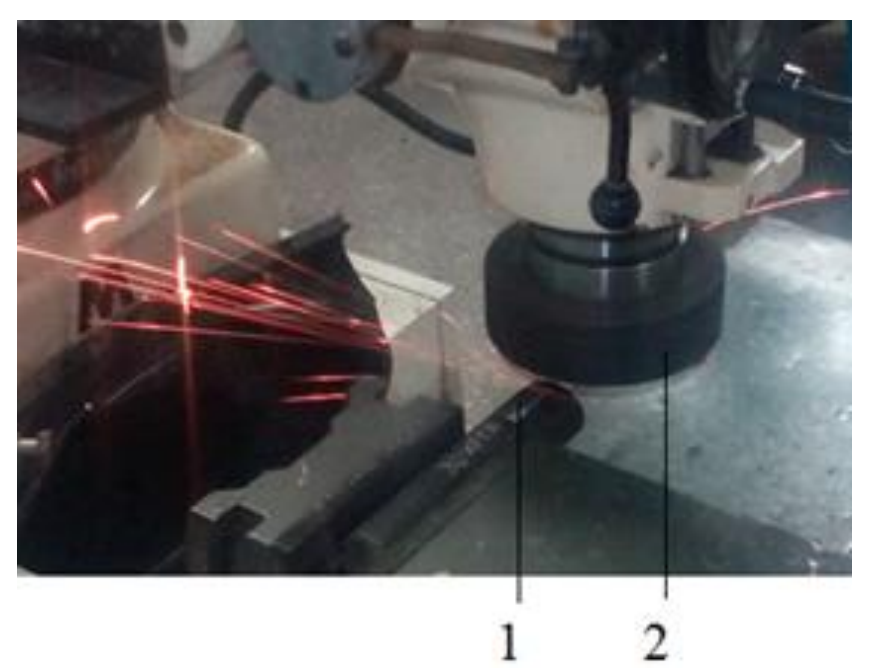

Fig. 1. The process of thermofrictional milling of 30CGSA steel. 
For further research, thermal processes occurring in the "cutting tool-workpiece" contact zone are of scientific and applied importance. Getting information about physical processes in various points of the cutting zone by experiments proves difficult due to the small size of the contact area of the cutting tool surface and the material being processed, the high speed of the process, and the constant relative motion between the cutting tool, the processed material and the chip formation process. In this regard, mathematical modelling of the thermofrictional milling can solve this problem through computer simulation. A similar problem was partially addressed in a number of papers $[7,8]$. However, they made only a general analysis of physical processes for the entire cutting insert. Using special programs, for example AnsysWB, enables to study the dynamics of processes occurring during cutting as well as take into account multiple-option technological solutions that may foster the process efficiency. The purpose of this study is to develop a methodology for determining the optimum technological parameters of cutting by thermofrictional milling with AnsysWB, when processing materials 40CN2MA, 30CGSA, Steel 45 and Steel 3sp.

\section{EXPERIMENT}

To build up the model, the following steps were performed: developing the CAD model of the cutting tool and the workpiece; introducing the physical characteristics of the cutting tool/workpiece materials; setting a type of contact between the surface of the cutting insert and the workpiece; specifying conditions for applying a finite element mesh. Physical properties of the processed materials and the cutting insert of the end mill (Hardox 450), used in the models, are presented in Table 1.

Table 1. Physical properties of the workpieces

\begin{tabular}{lcccccc}
\hline \hline Parameter & Unit & Hardox 450 & 40CN2 & 30CGS & St. 45 & St. 3sp \\
\hline Density, $\rho$ & $\mathrm{kg} / \mathrm{m}^{3}$ & 7850 & 7850 & 7850 & 7850 & 7850 \\
Young's modulus, $E$ & $\mathrm{GPa}$ & 215 & 215 & 215 & 215 & 215 \\
Poisson's ratio, $\nu$ & - & 0.3 & 0.3 & 0.3 & 0.3 & 0.3 \\
Specific heat conductivity, $c_{p}$ & $\mathrm{~J} /(\mathrm{kgC})$ & 495 & 477 & 466 & 469 & 434 \\
Thermal conductivity, $\lambda$ & $\mathrm{W} /(\mathrm{mC})$ & 37 & 39 & 39 & 79 & 61 \\
Initial temperature, $T_{t}$ & ${ }^{\circ} \mathrm{C}$ & 22 & 22 & 22 & 22 & 22 \\
Melting point, $T_{f}$ & ${ }^{\circ} \mathrm{C}$ & - & 1520 & 1030 & 1500 & 1500 \\
\hline
\end{tabular}

Johnson-Cook law is the most prevalent one, among various laws of material behaviour under large deformations. It takes into account adiabatic shear phenomena, caused by large plastic deformations and significant temperature gradients. This law specifies the dependence of stress $\sigma$ on the degree $\varepsilon(\%)$ and the strain rate as well as on the temperature $T$, and can be decomposed in a multiplicative form into three 
functions

$$
\sigma=\left(A+B \varepsilon^{n}\right)\left(1+C \frac{\ln \varepsilon}{\varepsilon_{0}}\right)\left(1-\left[\frac{T-T_{0}}{T_{f}-T_{0}}\right]\right) .
$$

The first factor describes the hardening phenomenon, the second one describes dynamic processes, the third one deals with the tempering phenomenon. The factor associated with hardening corresponds to the flow stress of a constant strain rate. A is a limit of elasticity of the material in question, $\mathrm{B}$ and $\mathrm{n}$ are linear and non-linear parameters of hardening, respectively.

The second factor is a multiplicative factor, characterizing the dynamic hardening of the material. It depends on the equivalent rate of plastic deformation. $\mathrm{C}$ is a strain rate sensitivity coefficient. Let us assume the initial strain rate equal to $1 \mathrm{~s}^{-1}$.

The third factor is a factor corresponding to the heat release phenomenon. $T_{0}$ is the initial temperature. The motion stress reduces along with the temperature and tends to zero at $T=T_{f}$, for temperatures in the interval between $T_{0}$ and the melting temperature $T_{f}$. At temperatures tending to the melting point, the motion stress is practically zero. Thus, $T_{0}$ is the temperature with respect to which the heat release mechanism is considered, and $m$ is the exponent of the given heat release. The parameters of the Johnson-Cook model for the workpiece materials are presented in Table 2.

Table 2. Parameters of the Johnson-Cook model for the workpieces [9]

\begin{tabular}{lccccc}
\hline \hline Workpieces & A, MPa & B, MPa & C & n & m \\
\hline 40CN2MA & 1579 & 1316 & 0.5 & 0.0028 & 0.85 \\
30CGSA & 1680 & 500 & 0.5 & 0.015 & 1.0 \\
St. 45 & 410 & 280 & 0.47 & 0.0037 & 1.1 \\
St. 3sp & 286 & 500 & 0.228 & 0.0171 & 0.917 \\
\hline
\end{tabular}

The formation and separation of chips in the model is based on the law of material fracture. It follows on from the method of "element erosion", which consists in the evolution of a crack, depending on the deformation stress conditions in the body of a workpiece. Application of this law includes two criteria. The first criterion characterizes the required degree of material fracture, and the second one is responsible for the evolution of a crack, its spread and elements separation from each other.

The adapted fracture criterion is interconnected with the Johnson-Cook motion law. The fracture is calculated for each element starting from the moment when

$$
\omega=\sum \frac{\Delta \bar{\varepsilon}}{\Delta \bar{\varepsilon}_{f}}
$$


where $\Delta \bar{\varepsilon}$ is an increment of resulting plastic deformation, $\Delta \bar{\varepsilon}_{f}$ is the resulting deformation of material fracture [10]. Fracture in a material starts at $\omega=1$.

The Johnson-Cook fracture model takes into account thermomechanical processes at large deformations. The equation of the resulting failure plastic strain is presented as follows:

$$
\bar{\varepsilon}_{f}=\left[D_{1}+D_{2} \exp \left(D_{3} \sigma^{*}\right)\right]\left[1+D_{4} \ln \bar{\varepsilon}^{*}\right]\left[1+D_{5} T^{*}\right],
$$

where $\sigma^{*}$ is a ratio of average stress $\left(\sigma_{m}\right)$ and resulting von Mises stress $(\bar{\sigma})$; $\bar{\varepsilon}^{*}$ is dimensionless degree of plastic deformation at an estimated strain rate and the limit characterizing the moment of sensitivity to $\dot{\varepsilon}_{0}$ strain rate. The dimensionless temperature coefficient $T^{*}$ can be written in the form

$$
T^{*}=\left(T-T_{0}\right) /\left(T_{f}-T_{0}\right),
$$

where $T_{f}$ is a material melting point; $T_{0}$ is the initial temperature; $D_{1}$ is the initial fracture deformation; $D_{2}$ is the exponential factor; $D_{3}$ is a factor of three-axis; $D_{4}$ is the strain rate factor; $D_{5}$ is the thermal factor [11].

The parameters of equation (3) of the workpiece fracture plastic strain are presented in Table 3.

Table 3. The parameters of the workpiece fracture plastic strain [9]

\begin{tabular}{lccccc}
\hline \hline Workpieces & $\boldsymbol{D}_{\mathbf{1}}$ & $\boldsymbol{D}_{\mathbf{2}}$ & $\boldsymbol{D}_{\mathbf{3}}$ & $\boldsymbol{D}_{\mathbf{4}}$ & $\boldsymbol{D}_{\mathbf{5}}$ \\
\hline 40CN2MA & 0.05 & 3.44 & -2.12 & 0.002 & 0.61 \\
30CGSA & 0.54 & 4.89 & -3.03 & 0.014 & 1.12 \\
St. 45 & 0 & 1.3 & -0.17 & 0.063 & 2.8 \\
St. 3sp & -0.8 & 2.1 & -0.5 & 0.002 & 0.61 \\
\hline
\end{tabular}

In case, when the fracture criterion is met, the fracture development criterion comes into effect. This criterion presents the level of energy $\mathrm{G}_{f}$, necessary for the development of a crack. After a crack occurs, the material behaviour is calculated by the relation of stress and displacement, but not those of stress and deformation. For the scientific study of the cutting process, it is necessary to obtain the following information: contact force in the contact zone and temperature distribution in the workpieces. A denser superposition of the finite element mesh allows to obtain more accurate results in modelling the physical processes, but it takes much time. Therefore, the relation between required accuracy and time constraints always prevails. In order to analyse physical processes in the cutting zone, we will show the workpiece surface in the cutting zone and the number them, as well. 

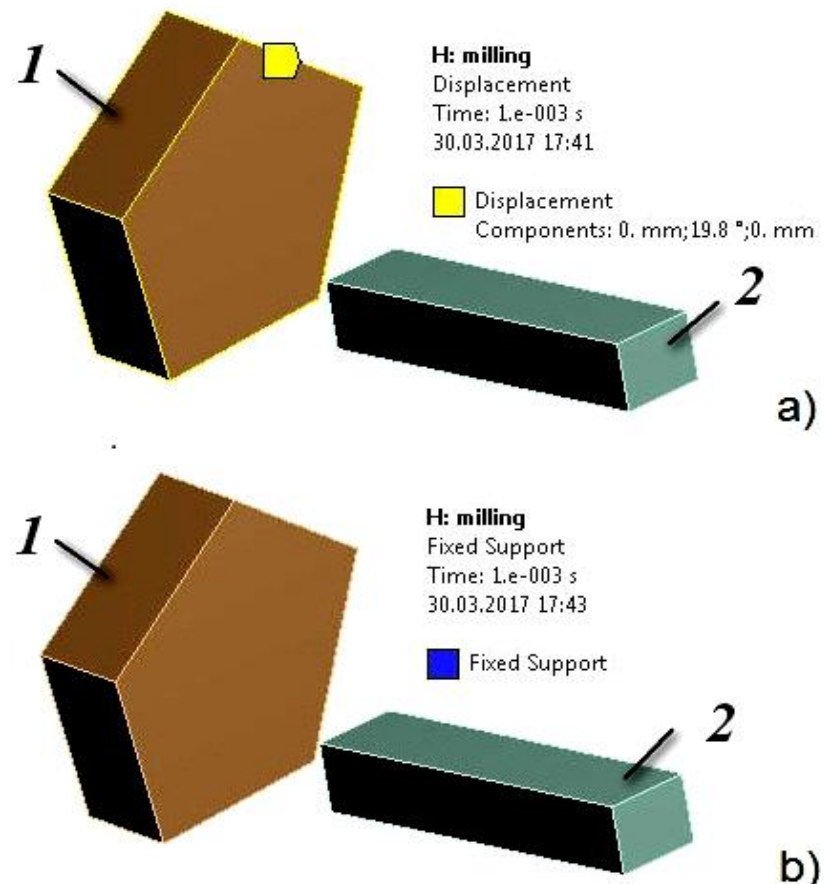

b)

Fig. 2. Conditions of supporting: a) of a tool insert, b) of a workpiece.

Figure 2 shows the conditions of supporting of the tool insert and the workpiece. The process of plane milling in Ansys WB is simulated, using the Explicit Dynamics module. This type of analysis is used for finite element simulation of mechanical processing, which is highly nonlinear. In the project tree in Connections, Contact

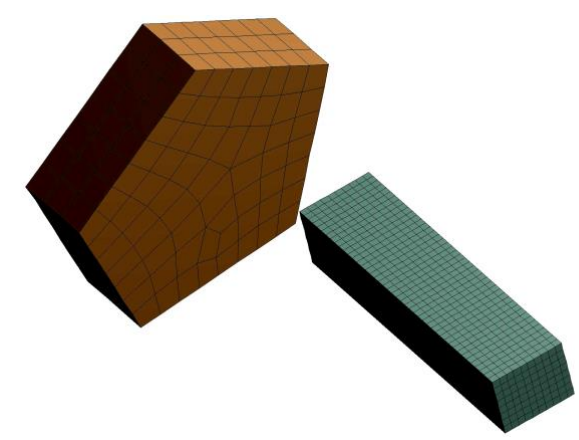

Fig. 3. Finite element mesh of the tool insert and the workpiece. 


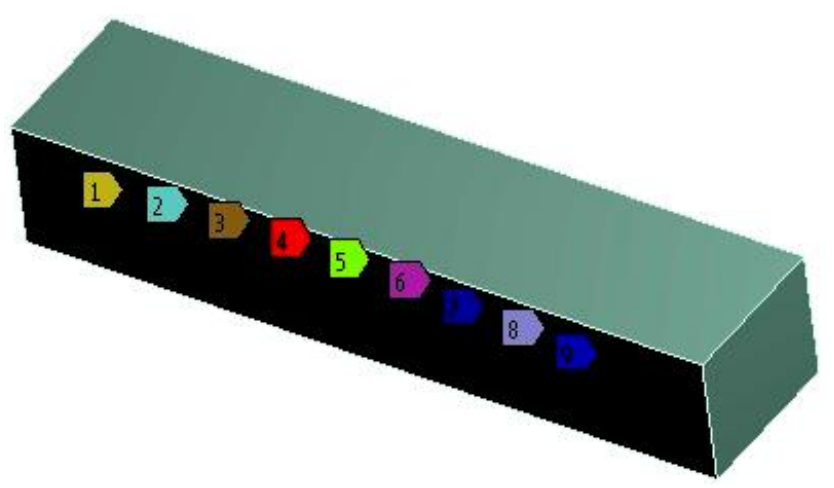

Fig. 4. Numbering of characteristic points on the workpiece surface in the thermofrictional milling zone.

frictional is used with a friction coefficient of 0.4. Boundary conditions are also imposed. Rotation of the milling cutter is simulated by the angle of rotation of one tool insert. One (1) object is a tool insert and it performs translational motion along the arc towards the workpiece 2, while the workpiece 2 possesses a fixed support.

Figure 3 shows the finite element mesh of the tool insert and a workpiece.

Figure 4 shows the numbering of characteristic points on the workpiece surface in the thermofrictional milling zone.

The treated workpiece surface is divided into uniformly located points for more

(a)

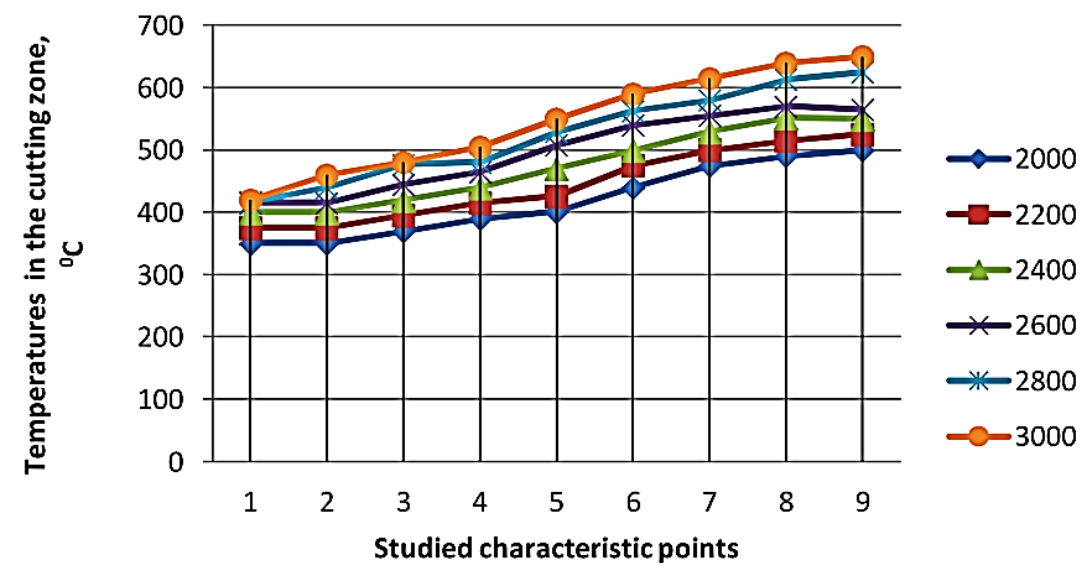

Fig. 5. Contact temperature during the processing of workpieces of various materials: (a) 40CN2MA; (b) 30CGSA; (c) St.45; (d) St.3sp ( $S=21.6 \mathrm{~mm} / \mathrm{min} ; n=2000,2200,2400$, 2600, 2800, $3000 \mathrm{rpm})$. 
(b)

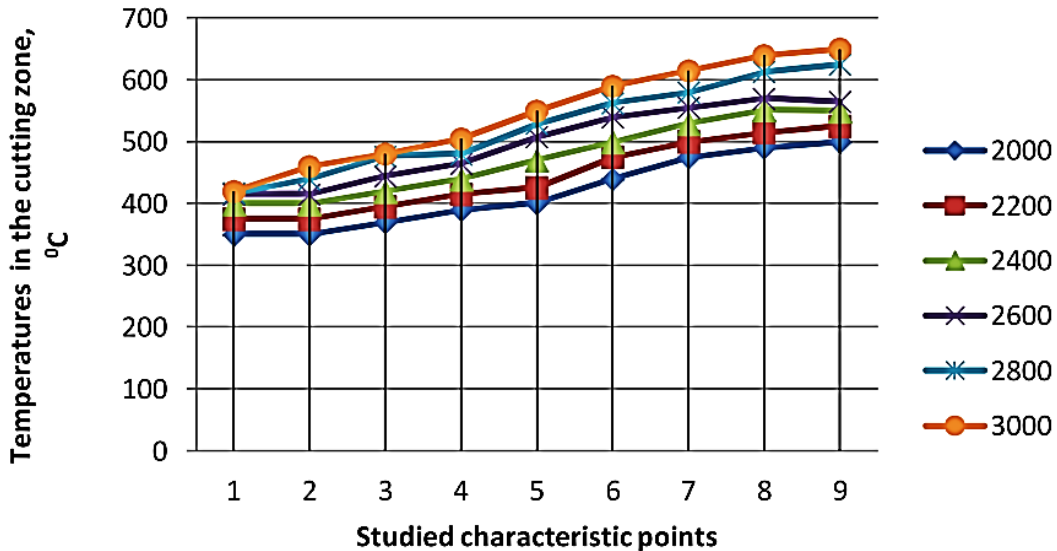

(c)

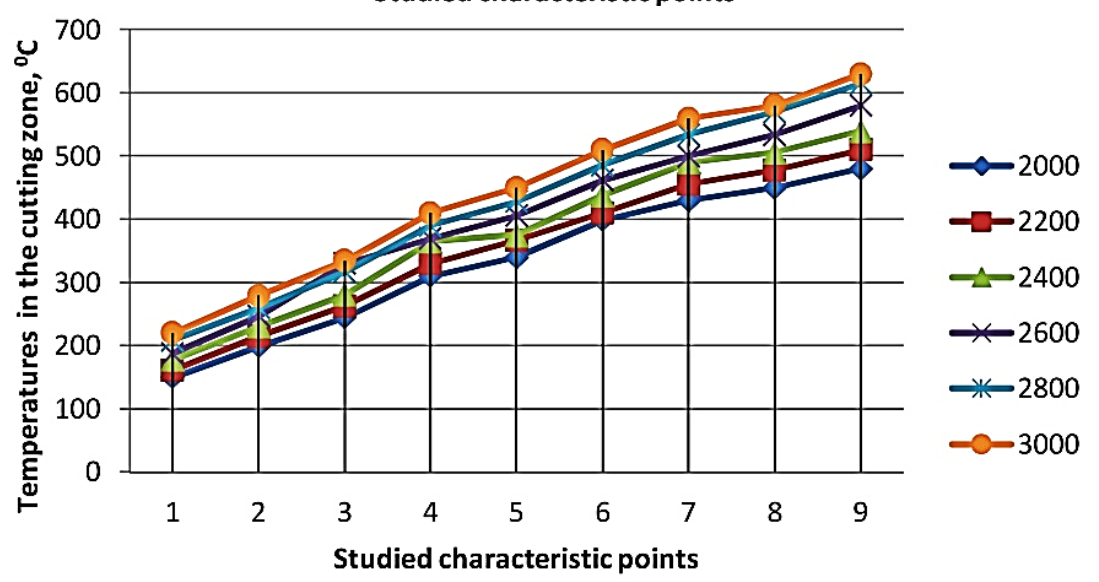

(d)

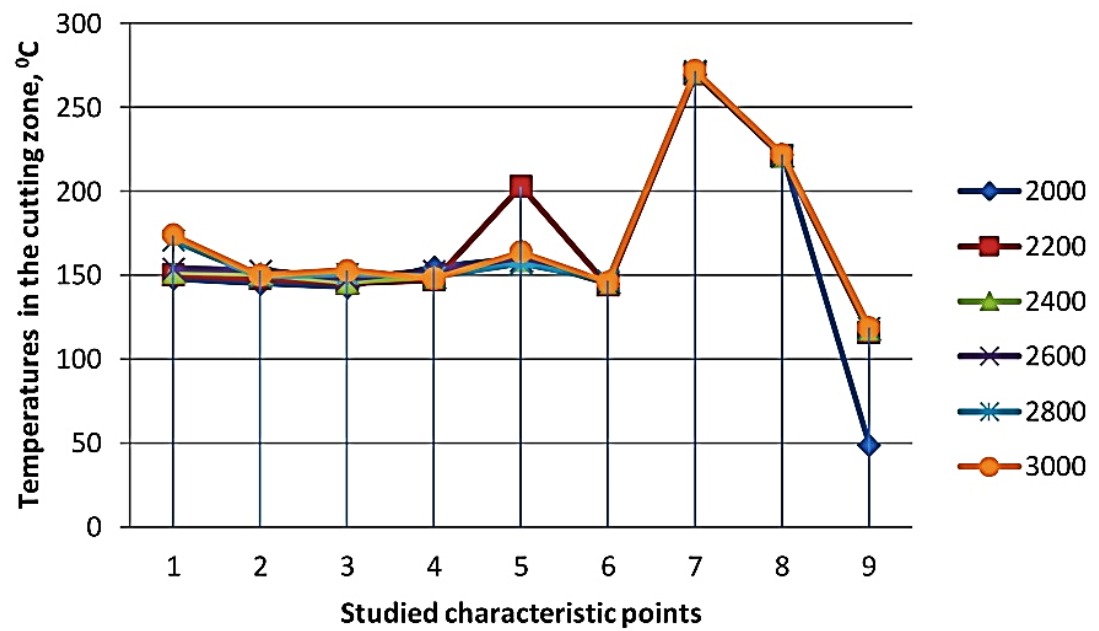


detailed information about the processes taking place in the "tool-workpiece" contact zone during thermofrictional milling, These characteristic points can be considered as a trace of cutting inserts making an arc-wise working stroke. We build the diagram of changes in the contact temperature from the tool rotation frequency in order to analyse physical processes in the cutting zones. Figure 5 shows the dependence of the contact temperature on the rotation speed, when processing workpieces of various materials.

As seen from the graphs, with an increase in the tool rotation frequency there is a temperature increase in the tool-worpiece contact. For the proposed method of milling, it is necessary to provide the temperature in the tool-workpiece contact close to the processed material's recrystallization temperature [5]. Let us consider the graph (Fig. 5, c) of the dependence of the contact temperature on the rotation frequency during the processing of St. 45 . For St. $45 T_{m}=1400^{\circ} \mathrm{C}$, and the recrystallization temperature is $T_{\mathrm{rec}} \approx 600^{\circ} \mathrm{C}$. It can be seen from the graph, that at the given processing modes, the temperature in the "tool-workpiece" contact does not exceed the recrystallization temperature. This confirms the optimality of the given processing modes.

This technique allows to determine the optimal cutting modes for thermofrictional milling for processing various materials, in particular steel 40CN2MA, 30CGSA, 45, 3 sp. In our research, we chose the criterion first proposed by prof. V. L. Kolmogorov from among a number of existing models of cutting fracture. In order to increase the calculations performance, a mathematical model was proposed, that used only two objects: a parallelepiped-shaped workpiece and a cutting insert in the form of a pentagonal prism. In addition, the work takes into account the friction coefficient between a cutting insert and a workpiece taken, equal to $0.4 \mathrm{~mm}$. We introduced the coordinates of nine characteristic points with the same interval in the local coordinate system in order to determine the temperature in the subcontact layer of the workpiece. As a result, the temperature values were obtained for different materials at the studied points, during the cutter speed variation. The research results showed the possibility of controlling thermal processes, during processing by choosing the optimum cutting modes

\section{CONCLUSIONS}

1. The developed AnsysWB-based computer model of the cutting process allowed studying the dynamics of thermal and physical processes, occurring during the processing. This technique also allows to determine the optimum cutting modes for thermofrictional milling for processing various materials.

2. The results of studying thermal processes in the "tool-workpiece" contact zone, 
during thermofrictional milling of various materials by an end mill equipped with HARDOX 450 steel-based plates, showed the possibility of controlling thermal processes by selecting the optimum cutting modes.

3. The results obtained (see Fig. 5) once again confirm the hypothesis of the cutting mechanism $[5,12]$ of low-speed thermofrictional processing.

\section{REFERENCES}

[1] Nechayev, K. N. Thermo Frictional Processing - perspective Technological Direction and Assembly of Parts., Tool and Technology, Spb. Mechanical Engineering Institute, 2005, No. 17-18, 157-162.

[2] Kushnazarov, I. K., K.T. Sherov, A. Goldenberg et AL. The Way of Cutting Metal Workpieces. // Patent No. 2738 UZ. Special Messenger No. 3, 1995, 33-34.

[3] Sherov, K. N., D. U. Alikulov, K. I. Imashevo et Al. The Method of Thermo Frictional Processing of the Flat and Construction of Friction Disk, Innovative Patent No. 22998 R K for invention 15.10.2010, bull. No. 10. P. 50.

[4] Sherov, K. T., T. M. Busauova. A. K. Sherov et AL. The Way of Thermo Frictional Cutting-hardening Processing Cylindrical Surfaces and Construction of Disk Friction, Innovative Patent No. 25649 RK for invention 16.04.2012, bull. No. 4.

[5] Kushnazarov, I. K., K.T. Sherov, R. R. Altynbayev. Technology of Thermo Frictional Milling with a High-cooled, High Technology in Machine Equipment: Collected Works NTU “XIII".- Kharkiv, 2007, bull. 2, No. 15, 134-139.

[6] Siknimbayev, M. R. The Perspective Technology of Manufacturing Basic Details. Germany: Publishing House Palmarium Aademi Publishing, 2013, 267 p.

[7] Sikhimbayev, M. R., M. M. Musayev, B. S. Donenbayev et Al. Mathematical Modelling of Temperature Field during Thermo Frictional Processing, University Proceedings. - Karaganda: Publishing House KSTU, 2016. - No. 1 (62), 27-33.

[8] Mazdubay, A. B., M. M. Musayev, B. S. Donenbayev. The Investigation of Stable Disk during Thermo Frictional Cutting using Software ANSIS, Ambassador of Kazakh Academy of Transport and Communication Named by M.Tynyshpaeva - Almaty, Publishing House KazATK, 2015, No. 4 (94), 35-43.

[9] Zhang, Y., T. Mabrouki, D. Nelias, Y Gong. FE-model for Titanium Alloy (Ti-6Al-4V) Cutting based on the Identification of Limiting Shear Stress at Tool-chip Interface. France, Springer-Verlag, 2010.

[10] Ducobu, F., E. Filippi, E. Riviere-Lorphevre. Modelisation de l'influence de la Profondeur de Coupe en Micro-coupe Orthogonale, 19eme Congres Frangais de Mecanique. Marseille, 2009, 47-49.

[11] BACARiA, J. L. Un Modele Comportemental et Transitoire Pour la Coupe des Metaux, Toulouse, 2001.

[12] Sherov, K.T. Hypothesis of Cutting with Circular Saws Friction, The materials of Scientific-theoretical and Technical Conference "ISTIQLOL-5". Mining, Metallurgy and Machine Building. Part 1. - Publishing House Navoi, 1996, 78-79. 